\title{
Comparison of gamma index based on dosimetric error and clinically relevant dose-volume index based on three- dimensional dose prediction in breast intensity-modulated radiation therapy
}

Akari Kaneko ${ }^{1,2^{*}}$, Iori Sumida ${ }^{1}$, Hirokazu Mizuno ${ }^{1}$, Fumiaki Isohashi ${ }^{1}$, Osamu Suzuki ${ }^{1}$, Yuji Seo ${ }^{1}$, Keisuke Otani ${ }^{1}$, Keisuke Tamari ${ }^{1}$ and Kazuhiko Ogawa ${ }^{1}$

\begin{abstract}
Background: Measurement-guided dose reconstruction has lately attracted significant attention because it can predict the delivered patient dose distribution. Although the treatment planning system (TPS) uses sophisticated algorithm to calculate the dose distribution, the calculation accuracy depends on the particular TPS used. This study aimed to investigate the relationship between the gamma passing rate (GPR) and the clinically relevant dosevolume index based on the predicted 3D patient dose distribution derived from two TPSs (XiO, RayStation).

Methods: Twenty-one breast intensity-modulated radiation therapy plans were inversely optimized using XiO. With the same plans, both TPSs calculated the planned dose distribution. We conducted per-beam measurements on the coronal plane using a 2D array detector and analyzed the difference in 2D GPRs between the measured and planned doses by commercial software. Using in-house software, we calculated the predicted 3D patient dose distribution and derived the predicted 3D GPR, the predicted per-organ 3D GPR, and the predicted clinically relevant dose-volume indices [dose-volume histogram metrics and the value of the tumor-control probability/ normal tissue complication probability of the planning target volume and organs at risk]. The results derived from $\mathrm{XiO}$ were compared with those from RayStation.

Results: While the mean 2D GPRs derived from both TPSs were $98.1 \%$ (XiO) and 100\% (RayStation), the mean predicted 3D GPRs of ipsilateral lung (73.3\% [XiO] and 85.9\% [RayStation]; $p<0.001)$ had no correlation with 2D GPRs under the $3 \%$ global $/ 3 \mathrm{~mm}$ criterion. Besides, this significant difference in terms of referenced TPS between $\mathrm{XiO}$ and RayStation could be explained by the fact that the error of predicted $\mathrm{V}_{5 \mathrm{~Gy}}$ of ipsilateral lung derived from $\mathrm{XiO}(29.6 \%)$ was significantly larger than that derived from RayStation $(-0.2 \% ; p<0.001)$.

Conclusions: GPR is useful as a patient quality assurance to detect dosimetric errors; however, it does not necessarily contain detailed information on errors. Using the predicted clinically relevant dose-volume indices, the clinical interpretation of dosimetric errors can be obtained. We conclude that a clinically relevant dose-volume index based on the predicted 3D patient dose distribution could add to the clinical and biological considerations in the GPR, if we can guarantee the dose calculation accuracy of referenced TPS.
\end{abstract}

\footnotetext{
* Correspondence: a.kaneko@radonc.med.osaka-u.ac.jp

${ }^{1}$ Department of Radiation Oncology, Osaka University Graduate School of

Medicine, 2-2 Yamada-oka, Suita 565-0871, Osaka, Japan

${ }^{2}$ Department of Radiology, Suita Tokushukai Hospital, 21-1 Senrioka-nishi,

Suita 565-0814, Osaka, Japan
}

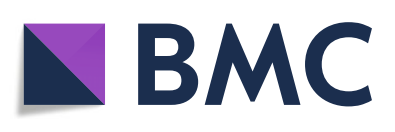

(c) The Author(s). 2019 Open Access This article is distributed under the terms of the Creative Commons Attribution 4.0 International License (http://creativecommons.org/licenses/by/4.0/), which permits unrestricted use, distribution, and reproduction in any medium, provided you give appropriate credit to the original author(s) and the source, provide a link to the Creative Commons license, and indicate if changes were made. The Creative Commons Public Domain Dedication waiver (http://creativecommons.org/publicdomain/zero/1.0/) applies to the data made available in this article, unless otherwise stated. 


\section{Background}

Patient-specific quality assurance (QA) is essential in intensity-modulated radiation therapy (IMRT). No current treatment planning system (TPS) completely considers dose uncertainties due to the beam-delivery system in IMRT, for example, source distribution, leaf thickness of multileaf collimators (MLCs), leakage, tongue-and-groove structures, and the dosimetric effective MLC offset [1]. The gamma passing rate (GPR), introduced by Low et al. [2], conveniently combines the dose difference and the distance to agreement by considering the difference between the planned and measured dose distributions. The GPR, which is widely used for patient-specific QA, is recommended by the American Association of Physicists in Medicine Task Group (AAPM TG) 119 [3] and 218 [4]. Although conflicting findings about the effectiveness of the GPR regarding error detection have often been reported [5-7], the GPR still provides evidence of the dose error between planned and delivered dose distributions that is sufficiently strong to lead to the approval of clinical treatments [3, 4].

Measurement-guided dose reconstruction (MGDR) [7-9] using commercial 3DVH software (Sun Nuclear Corporation, Melbourne, FL, USA) [10] has been used to predict the delivered 3D dose distribution, including potential dose error during beam delivery. On the basis of the differences between the predicted and planned distributions, various clinically relevant dose-volume indices have been discussed, such as per-organ dose-volume histogram (DVH) metrics, tumor-control probability/normal tissue complication probability (TCP/NTCP) [11]. With regard to the relationship between GPR and the predicted DVH metrics, Nelms et al. [7] demonstrated that the GPR is not correlated with dose errors in organs at risk (OARs).

Most previous studies of MGDR for QA used commercial software, such as COMPASS (IBA Dosimetry, Schwarzenbruck, Germany) [12] and 3DVH. These systems require additional beam modeling or re-calculation of the dose distribution, which generates extra uncertainty in addition to the uncertainty of dose calculation of the original plan. To overcome this issue, we proposed a simplified MGDR procedure that uses the in-house software developed in our previous work [13-16], which only adds a local error distribution along with the incident photon flux passing through the beam's eye view.

In addition, several studies of MGDR [11, 17] have demonstrated that the results of the predicted DVH metrics or TCP/NTCP change depending on machine delivery errors and TPS beam modeling. Although the current methods to calculate the TPS dose distribution are sophisticated, elements constituting the beam model and the type and number of modeling parameters are not the same for each TPS. For example, the XiO TPS (ELEKTA Instrument AB, Stockholm, Sweden) uses a single-photon-source model [18], whereas the RayStation TPS (RaySearch Medical Laboratories AB, Stockholm, Sweden) uses a dual-source photon-beam model [19], which comprises a primary-photon-source target and an additional component serving as an extra-focal photon source (e.g., the primary collimator and flattening filter) [20, 21]. Furthermore, although $\mathrm{XiO}$ has a few beam modeling parameters that users can optimize, RayStation has many [22-24].

Radiation pneumonitis has been indicated after irradiation for breast-cancer radiotherapy [25]. In breast IMRT, the dose absorbed in the lung should be considered because the mean dose in the lung for breast IMRT typically exceeds that for three-dimensional conformal radiotherapy [26]. Because the TCP/NTCP values are associated with the TPS-calculated doses, several studies have reported that calculated NTCP values for the lung, combined with the inhomogeneity corrections for dose calculations, are sensitive to differences in the calculation algorithms $[27,28]$.

The present study aims to verify the usefulness of the predicted clinically relevant dose-volume index (DVH metrics and TCP/NTCP values) based on the predicted $3 \mathrm{D}$ patient dose distribution by comparison with the $2 \mathrm{D}$ GPR, the predicted 3D GPR, and the predicted per-organ 3D GPR using the in-house MGDR software in breast IMRT. In addition, the issue that indices derived from the predicted $3 \mathrm{D}$ patient dose distribution change significantly depending on the accuracy of the referenced TPS is also demonstrated.

\section{Methods}

\section{Treatment planning and delivery}

This study is based on 21 clinically approved left-sided breast IMRT plans, whose parameters are summarized in Table 1. All patients were treated with an ARTISTE (Siemens Medical Systems, Concord, USA) using a 6-MV photon beam and 160 MLCs with a 5-mm leaf width. Computed tomography (CT) image acquisition and

Table 1 Description of 21 plans

\begin{tabular}{ll}
\hline & Value (range) \\
\hline \# Beams & 2 (all plans) \\
Minimum segment size & $2 \times 2 \mathrm{~cm}^{2}$ (all plans) \\
Minimum monitor units (MUs) per segment & $5 \mathrm{MU}$ (all plans) \\
Mean (range) \# segments & $35 \pm 7$ (19-46) \\
Mean (range) equivalent square side (cm) & $8.6 \pm 0.8(7.4-10.2)$ \\
Dose-volume constraint of PTV: $D_{95 \%}$ & $\geq 95 \%(47.5 \mathrm{~Gy})$ \\
Dose-volume constraint of ipsi. & $<15 \%$ (per protcol), <20\% \\
lung: $V_{20 G y}$ & (variation acceptable) \\
Dose-volume constraint of heart: $D_{\text {mean }}$ & $<4$ Gy \\
\hline
\end{tabular}

$\mathrm{D}_{95 \%}$ : dose coverage $95 \%$ volume, ipsi.: ipsilateral, $\mathrm{V}_{20 \mathrm{~Gy}}$ : volume receiving at least $20 \mathrm{~Gy}, \mathrm{D}_{\text {mean }}$ : dose coverage mean volume 
radiation treatment were performed under free breathing. All IMRT plans involved two tangentially opposed beams in fixed-gantry step-and-shoot delivery using the $\mathrm{XiO}$ TPS. Gantry and collimator angles were selected to avoid contralateral breast irradiation and minimize exposure to the ipsilateral lung. The prescription dose was $50 \mathrm{~Gy}$ in 25 fractions to $\mathrm{D}_{50 \%}$ of the planning target volume (PTV). The PTV and OARs were contoured, and the dose constraints were assigned based on the protocol of the Radiation Therapy Oncology Group 1005 [29]. The first $5 \mathrm{~mm}$ of tissue under the skin was omitted from the PTV. Based on target prescription and dose-volume constraints, IMRT plans were inversely optimized in $\mathrm{XiO}$.

The Digital Imaging and Communications in Medicine-Radiotherapy (DICOM-RT) CT image, plan, and structure files of all 21 breast IMRT plans were exported from $\mathrm{XiO}$ to RayStation. The delivery monitor unit and shape of segments were exactly the same for all fields for each TPS plan. Both TPSs used a superposition algorithm with a 2-mm grid size to calculate the dose distribution. Note that $\mathrm{XiO}$ and RayStation were approved by commissioning tests defined by AAPM TG 53 [30] and ESTRO Booklet 7 [31] and are used in our institution of clinical radiotherapy.

\section{Patient-specific QA based on dosimetric error}

For patient-specific QA based on dosimetric error, 2D GPRs were calculated from the measured and planned dose distributions obtained by each TPS. Per-beam measurements were performed for all plans on the coronal plane (depth $5 \mathrm{~cm}$, source-to-axial distance $100 \mathrm{~cm}$ ) and at zero gantry angle using MapCHECK (Sun Nuclear Corporation, Melbourne, FL, USA), of which spatial resolution was $5-\mathrm{mm}$ with a detector density. 2D GPRs were analyzed by the commercially supplied software included with MapCHECK with the criteria of 3\% global/ $3 \mathrm{~mm}, 3 \%$ global $/ 2 \mathrm{~mm}$, and $2 \%$ global $/ 2 \mathrm{~mm}$ with a lower threshold of $10 \%$. We adopted multiple gamma criteria in this study to understand the cause and impact of error by comparing with multiple sensitivity as mentioned in AAPM TG 218 [4].

\section{Patient-specific QA based on predicted 3D patient dose distribution}

For patient-specific QA based on the predicted 3D patient dose distribution, we calculated the predicted 3D GPR, the predicted per-organ 3D GPR, and the predicted clinically relevant dose-volume indices (DVH metrics and the value of TCP/NTCP of the PTV and OARs) using the in-house MGDR software application [14].

The procedure for deriving these indices is shown in Fig. 1. First, we created a per-beam planar relative dose error map with a 5-mm grid resolution, which is based on the per-beam planned 2D dose by each TPS and the measured 2D dose by MapCHECK. Second, the per-beam tentative predicted $3 \mathrm{D}$ patient dose distribution was reconstructed from the per-beam planar relative dose error map and the planned patient 3D dose distribution by each TPS with a 2-mm calculation grid.

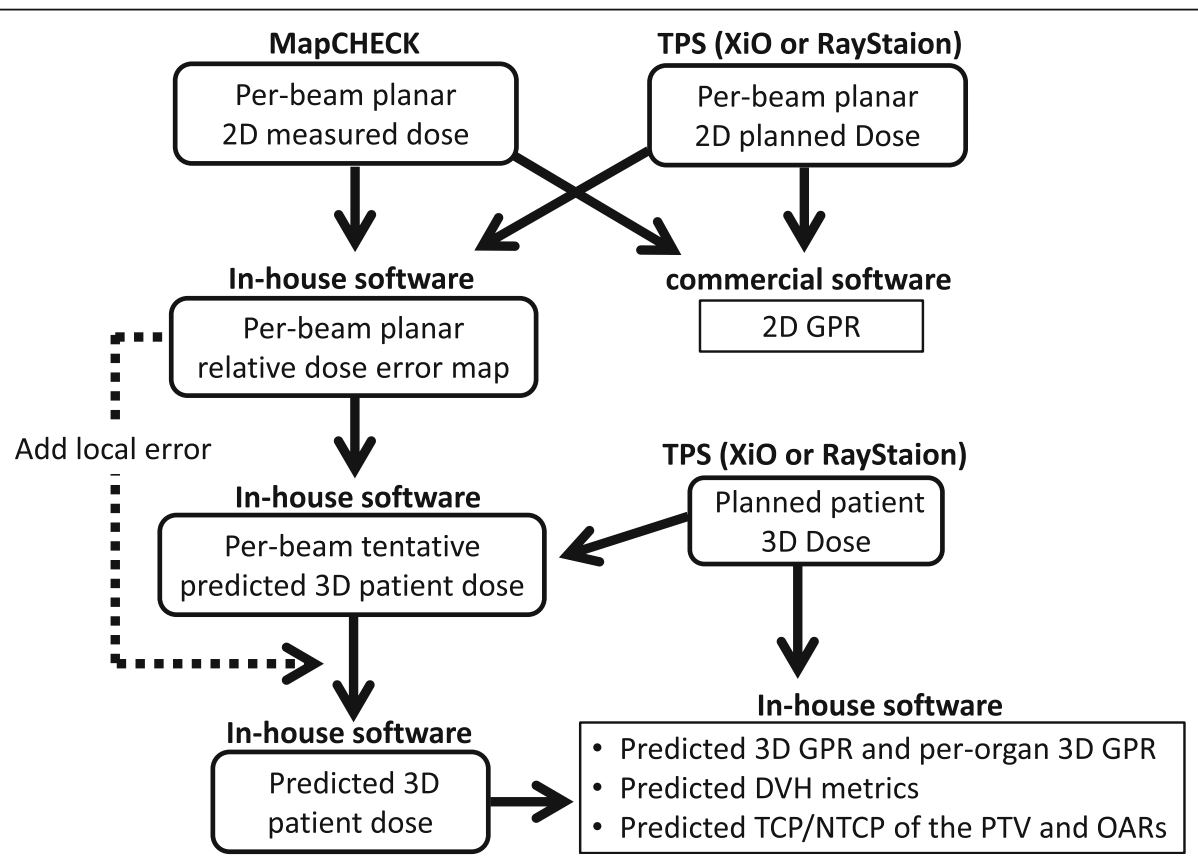

Fig. 1 The procedure for deriving the predicted 3D GPR, the predicted per-organ 3D GPR, and the predicted clinically relevant dose-volume indices (DVH metrics and the value of TCP/NTCP of the PTV and OARs) 
Third, the ray from the source to each tentative 3D dose grid was defined. The local error of intersection point between the ray and the per-beam planar relative dose error map located on the isocenter plane was obtained by the linear interpolation. Thus, the per-beam tentative predicted 3D dose grid was given the local error along the ray. Fourth, the predicted 3D patient dose distribution was obtained by summation of the per-beam tentative predicted 3D dose grid [14]. Typical axial planes of the predicted 3D patient dose distribution and dose-difference distributions with the planned 3D dose distribution are shown in Fig. 2.

Finally, we calculated the predicted 3D GPR and the predicted per-organ 3D GPR with criteria of 3\% global $/ 3$ $\mathrm{mm}, 3 \% \mathrm{global} / 2 \mathrm{~mm}$, and $2 \%$ global $/ 2 \mathrm{~mm}$ with a lower threshold of $3 \%$ and the predicted clinically relevant dose-volume indices by comparison with the predicted 3D dose distribution and the planned patient 3D dose distribution of each TPS. The results derived from the $\mathrm{XiO}$ TPS were compared with those from the RayStation TPS.

The TCP is calculated using Niemierko's EUD-based model [32]:

$$
\operatorname{TCP}\left(D_{i}\right)=\frac{1}{1+\left(\frac{T C D_{50}}{D_{i}}\right)^{4 \gamma_{50}}},
$$

where $\mathrm{D}_{i}$ is the original or predicted dose of the $i$-th voxel, $\mathrm{TCD}_{50}$ is the dose required for a TCP of $50 \%$, and $\gamma_{50}$ is the slope of the normalized tumor dose-response curve at $\mathrm{TCD}_{50}$.

The NTCP is calculated using the relative seriality model [33, 34]:

$$
\begin{aligned}
\mathrm{NTCP} & =\left[1-\prod_{i=1}^{M}\left[1-P\left(D_{i}\right)^{S}\right]^{\Delta v_{i}}\right]^{\frac{1}{s}}, \\
& \text { where } P\left(D_{i}\right)=2^{-\exp \left[\operatorname{er}\left(1-\left(D_{i} / D_{50}\right)\right)\right]} .
\end{aligned}
$$

The $P\left(D_{i}\right)$ is the probability of no cell surviving, and $s$ is the generalized parameter describing relative seriality. The fractional volume, $\Delta v_{i}$, at doses $D_{i}$ is most conveniently obtained from the $M$ bins of the differential DVH for the organ in question. $D_{50}$ is the total dose required for a NTCP of $50 \%$, and $\gamma$ is the slope of the maximum normalized normal tissue dose-response curve. Table 2 summarizes the resource parameters for calculating the TCP/NTCP.

\section{Statistical analysis}

The normal distribution of the data was determined using the Shapiro-Wilk test. Depending on the results, data were compared using the two-tailed paired $t$-test or the Wilcoxon signed-rank test, and the correlation was determined using Pearson's or Spearman's correlation coefficient, R. A $p$ value $<0.05$ was considered statistically significant. All statistical analyses were performed using the software R (The R Foundation for Statistical Computing, Vienna, Austria).

\section{Results}

2D GPR and predicted 3D GPR

The top of Table 3 shows the mean 2D GPRs obtained by per-beam planar dose analyses. Regarding the clinical decision, because all plans were planned by TPS XiO, these
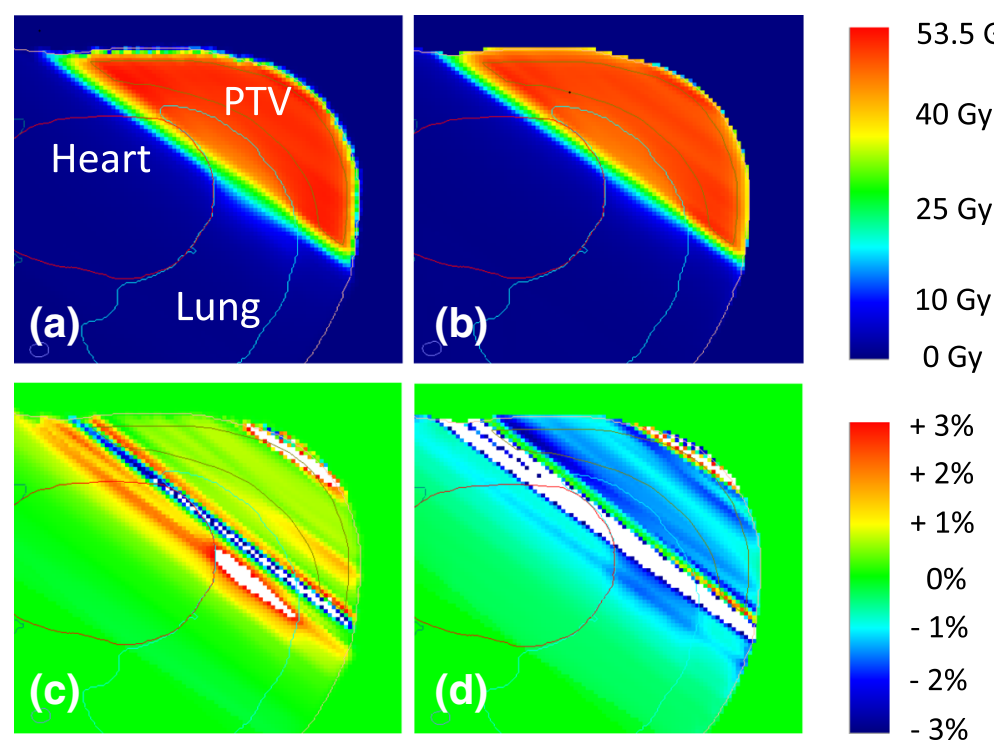

Fig. 2 Predicted dose and dose-difference distributions on a typical axial plane. (a) The predicted dose distribution planes derived from XiO TPS planned dose and (b) RayStation TPS planned dose. Dose-difference distribution (predicted versus planned dose) obtained by (c) XiO and (d) RayStation 
Table 2 Parameters for calculating TCP/NTCP

\begin{tabular}{|c|c|c|c|c|c|c|}
\hline Structure & Endpoint & Tissue-specific parameter & $\mathrm{TCD}_{50} / \mathrm{D}_{50}(\mathrm{~Gy})$ & $\gamma_{50} / Y$ & s & Ref. \\
\hline PTV & Local control & -7.2 & 30.89 & 1.3 & & {$[37,38]$} \\
\hline Lung & Pneumonitis & 1 & 26.16 & 0.973 & 0.012 & [28] \\
\hline Heart & Late cardiac mortality & 3 & 52.3 & 1.28 & 1 & [39] \\
\hline
\end{tabular}

$\mathrm{TCD}_{50}$ : dose required for $50 \%$ probability of tumor control, $\mathrm{D}_{50}$ : dose required for $50 \%$ probability of normal tissue complication, $\gamma_{50}$ : slope of normalized tumor dose-response curve at $\mathrm{TCD}_{50}, \gamma$ : slope of maxixum normalized normal tissue dose-response curve, s: relative seriality parameter

were verified with the $\mathrm{XiO}$ planned dose and accepted with criteria of 2 D GPRs $95 \%$ or over under $3 \% / 3 \mathrm{~mm}$ criterion. A few plans in which 2D GPRs were less than 95\% were considered clinically acceptable. The mean 2D GPRs from RayStation were $100 \%$ under the $3 \% / 3 \mathrm{~mm}$ criterion, which were significantly higher $(p<0.001)$ than those from $\mathrm{XiO}(98.1 \%)$. Under the $3 \% / 2 \mathrm{~mm}$ and $2 \% / 2 \mathrm{~mm}$ criteria, a similar tendency was also seen.

The bottom of Table 3 shows the predicted 3D GPRs. Under all criteria, the mean predicted 3D GPRs for RayStation were significantly higher $(p<0.001)$ than those for XiO. In addition, each predicted 3D GPR was lower than 2D GPR under all criteria. For example, the predicted 3D GPRs for $\mathrm{XiO}$ under 3\%/3 mm were lower than the 2D GPRs for $\mathrm{XiO}$ under $3 \% / 3 \mathrm{~mm}$.

\section{The predicted per-organ 3D GPR}

Figure 3 shows the correlations between the predicted per-organ 3D GPRs and 2D GPRs. The predicted 3D GPRs of PTV, ipsilateral lung, and heart do not correlate with the 2D GPRs except two datasets (the predicted 3D GPRs of PTV under $3 \% / 2 \mathrm{~mm}$ derived from $\mathrm{XiO}$ and the predicted 3D GPRs of ipsilateral lung under $2 \% / 2 \mathrm{~mm}$

Table 3 Summary of the GPR results

\begin{tabular}{|c|c|c|c|}
\hline & $\mathrm{XiO}$ & RayStation & \\
\hline & Mean \pm SD (range) & Mean \pm SD (range) & $P$ value \\
\hline \multicolumn{4}{|l|}{ 2D GPR } \\
\hline \multirow[t]{2}{*}{$3 \% / 3 \mathrm{~mm}$} & $98.1 \pm 1.9$ & $100.0 \pm 0.1$ & $p<0.001$ \\
\hline & $(93.5-100)$ & $(99.5-100)$ & \\
\hline \multirow[t]{2}{*}{$3 \% / 2 \mathrm{~mm}$} & $95.2 \pm 2.9$ & $99.8 \pm 0.5$ & $p<0.001$ \\
\hline & (86.5-99.0) & $(97.7-100)$ & \\
\hline \multirow[t]{2}{*}{$2 \% / 2 \mathrm{~mm}$} & $93.3 \pm 3.7$ & $99.5 \pm 0.8$ & $p<0.001$ \\
\hline & $(82.4-98.7)$ & $(95.7-100)$ & \\
\hline \multicolumn{4}{|c|}{ predicted 3D GPR } \\
\hline \multirow[t]{2}{*}{$3 \% / 3 \mathrm{~mm}$} & $91.3 \pm 1.6$ & $93.9 \pm 1.0$ & $p<0.001$ \\
\hline & $(88.6-94.5)$ & $(91.8-96.2)$ & \\
\hline \multirow[t]{2}{*}{$3 \% / 2 \mathrm{~mm}$} & $85.9 \pm 2.3$ & $91.2 \pm 1.1$ & $p<0.001$ \\
\hline & $(82.2-91.0)$ & $(89.0-93.5)$ & \\
\hline \multirow[t]{2}{*}{$2 \% / 2 \mathrm{~mm}$} & $82.1 \pm 3.5$ & $89.5 \pm 1.8$ & $p<0.001$ \\
\hline & $(77.2-87.8)$ & $(86.1-92.3)$ & \\
\hline
\end{tabular}

2D GPR averaged for all applicable fields. The predicted 3D GPR derived from each TPS (XiO or RayStaion) derived from RayStation). In addition, these datasets were in moderate correlation as shown in Fig. 3.

Regarding the referred difference in TPS, the mean predicted 3D GPRs of ipsilateral lung derived from RayStation was significantly higher than those from $\mathrm{XiO}$ under all criteria $(p<0.001)$, whereas the mean predicted 3D GPRs of PTV derived from RayStation were significantly lower than those from $\mathrm{XiO}$ under the $3 \% / 3 \mathrm{~mm}$ and $3 \% / 2 \mathrm{~mm}$ criteria $(p<0.001)$ (Table 4$)$.

\section{The predicted clinically relevant dose-volume indices}

Table 5 shows the mean predicted clinically relevant dose-volume indices. Figure 4 shows the mean deviations between the predicted and planned clinically relevant dose-volume indices. The mean deviations between the predicted and planned $\mathrm{V}_{5 \mathrm{~Gy}}$ and NTCP of ipsilateral lung derived from $\mathrm{XiO}\left(\mathrm{V}_{5 \mathrm{~Gy}}: 29.6 \%\right.$, NTCP: 9.2\%) were significantly larger than those derived from RayStation $\left(\mathrm{V}_{5 \mathrm{~Gy}}:-0.2 \%\right.$, NTCP: $-3.0 \%$; $p<0.001)$, whereas the deviations between the predicted and planned $\mathrm{V}_{20 \mathrm{~Gy}}$ of ipsilateral lung derived from RayStation and $\mathrm{XiO}$ showed no significant difference. Besides, the deviations between the predicted and planned PTV $\mathrm{D}_{95 \%}$ and the TCP of the PTV derived from $\mathrm{XiO}$ were significantly better than those derived from RayStation $(p<0.001)$.

\section{Discussion}

This study compares the 2D GPRs, the predicted 3D GPRs, the predicted per-organ 3D GPRs, and the predicted clinically relevant dose-volume indices derived from the two TPSs. The mean 2D GPRs and the mean predicted 3D GPRs from RayStation are higher than those from XiO (Table 3). The 2D GPR would be satisfactory for patient-specific QA with regard to evaluation of the dosimetric accuracy of the planned and measured data.

However, as several researchers have pointed out, the 2D GPR does not necessarily detect clinically significant dosimetric error, such as the dose deviation of OARs [5-7]. As shown in Table 4 and Fig. 3, under $3 \% / 3 \mathrm{~mm}$ criterion, the 2D GPRs do not correlate with the predicted per-organ 3D GPRs. Even under stricter criteria (i.e., $3 \% / 2 \mathrm{~mm}$ or $2 \% / 2 \mathrm{~mm}$ ), the $2 \mathrm{D}$ GPRs do not necessarily correlate with the predicted per-organ 3D GPRs.

Moreover, although the predicted per-organ 3D GPRs indicated an existence of a predicted error, it is difficult 

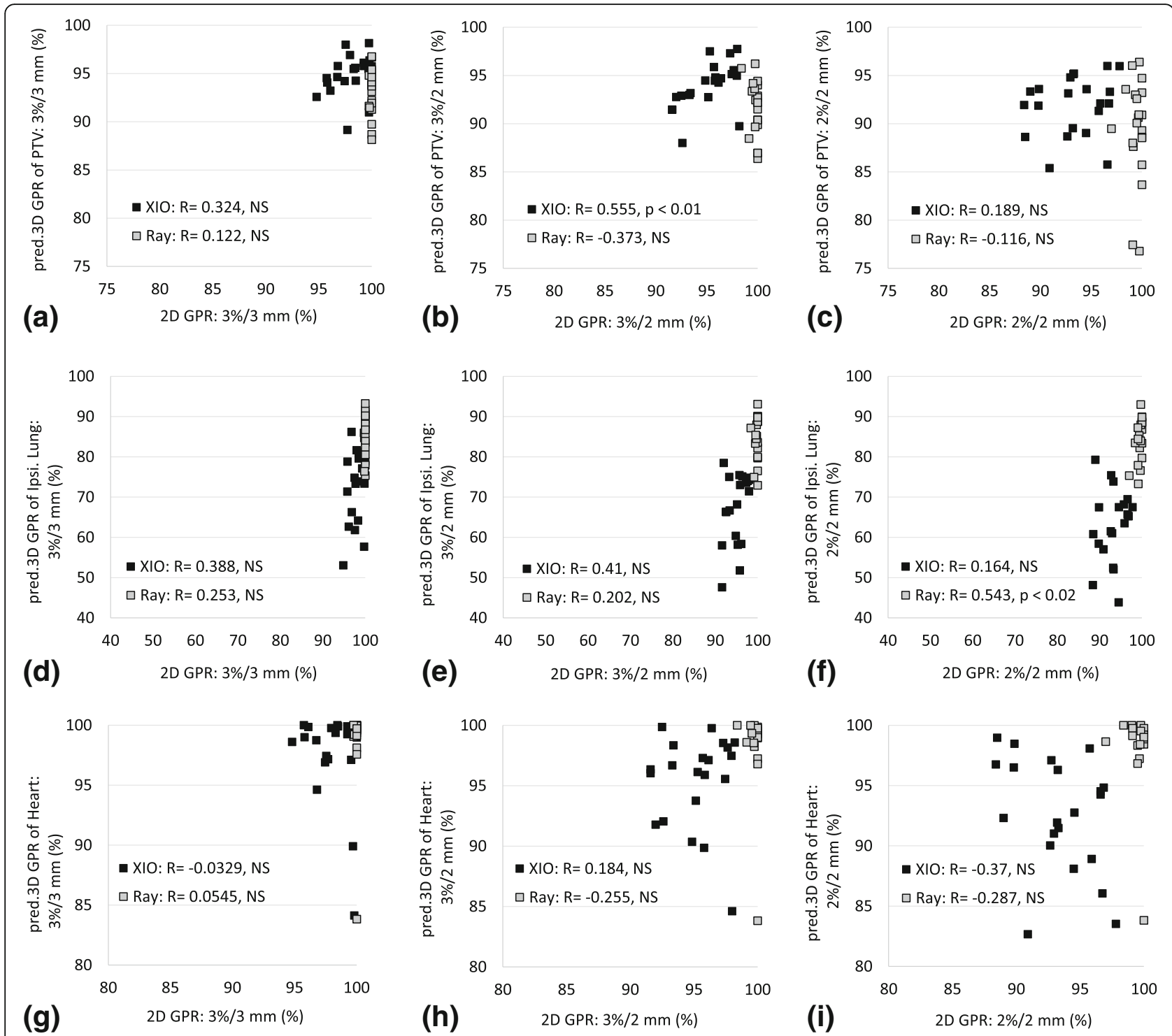

Fig. 3 Correlation between 2D GPR and predicted per-organ 3D GPR: (a), (b), (c) PTV, (d), (e), (f) ipsilateral lung, and (g), (h), (i) heart. "R" denotes the Pearson correlation coefficient or Spearman Rank-Order Correlation coefficient. Pred. $=$ Predicted. Ray $=$ RayStation. Ipsi. $=$ Ipsilateral.

NS = not significant

to understand the influence of such an error in a clinical context. The deviation between the predicted and planned clinically relevant dose-volume indices helps in this understanding, as shown in Table 5 and Fig. 4.

In addition, the predicted 3D GPRs, the predicted per-organ 3D GPRs, and the predicted clinically relevant dose-volume indices derived from the two TPSs have significant differences (Tables 3,4 and 5 and Figs. 3 and 4), which implies that the predicted 3D dose distribution is affected by the accuracy of the TPS model. With regard to the heart, it is difficult to ascertain the effect of the different TPSs in predicted 3D dose distributions because the relative volume of the heart is less than that of other organs in all plans.
Regarding the open-beam profile $\left(6 \mathrm{MV}, 10 \times 10 \mathrm{~cm}^{2}\right)$ for both TPSs, Table 6 and Fig. 5 show the beam profile measured using a 3D water tank and the analysis results calculated by $\mathrm{XiO}$ and RayStation. The beam profile was divided into three regions and analyzed. The central region is the central part and the flattened area of the profile $[80 \%$ or over of the central beam axis $(\mathrm{CAX})]$. The penumbra region represents the field edge with a rapid dose fall-off (between 20 and $80 \%$ of the CAX). The out-of-field region is outside the radiation field (up to $20 \%$ of the CAX). According to the Venselaar [35] formula, the percentage difference between the calculated and measured data is defined as follows: 
Table 4 Summary of predicted per-organ 3D GPR derived from each TPS (XiO or RayStaion)

\begin{tabular}{|c|c|c|c|}
\hline \multirow{2}{*}{$\begin{array}{l}\text { predicted } \\
\text { per-organ } \\
\text { 3D GPR }\end{array}$} & \multirow{2}{*}{$\begin{array}{l}\text { XiO } \\
\text { Mean } \pm \text { SD (range) }\end{array}$} & \multirow{2}{*}{$\begin{array}{l}\text { RayStation } \\
\text { Mean } \pm \text { SD (range) }\end{array}$} & \multirow[t]{2}{*}{$P$ value } \\
\hline & & & \\
\hline \multicolumn{4}{|l|}{ PTV } \\
\hline \multirow[t]{2}{*}{$3 \% / 3 \mathrm{~mm}$} & $94.9 \pm 2.1$ & $93.1 \pm 2.4$ & \multirow[t]{2}{*}{$p<0.001$} \\
\hline & $(89.2-98.2)$ & (88.2-96.8) & \\
\hline \multirow[t]{2}{*}{$3 \% / 2 \mathrm{~mm}$} & $93.9 \pm 2.4$ & $91.6 \pm 2.8$ & \multirow[t]{2}{*}{$p<0.001$} \\
\hline & (88.0-97.8) & $(86.4-96.2)$ & \\
\hline \multirow[t]{2}{*}{$2 \% / 2 \mathrm{~mm}$} & $91.9 \pm 3.1$ & $89.4 \pm 5.2$ & \multirow[t]{2}{*}{ Not Significant } \\
\hline & (85.4-96.0) & $(76.8-96.4)$ & \\
\hline \multicolumn{4}{|l|}{ Ipsilateral lung } \\
\hline \multirow[t]{2}{*}{$3 \% / 3 \mathrm{~mm}$} & $73.3 \pm 9.1$ & $85.9 \pm 5.0$ & \multirow[t]{2}{*}{$p<0.001$} \\
\hline & $(53.1-86.2)$ & (75.4-93.3) & \\
\hline \multirow[t]{2}{*}{$3 \% / 2 \mathrm{~mm}$} & $67.7 \pm 8.7$ & $84.6 \pm 5.4$ & \multirow[t]{2}{*}{$p<0.001$} \\
\hline & $(47.6-78.5)$ & $(73.0-93.1)$ & \\
\hline \multirow[t]{2}{*}{$2 \% / 2 \mathrm{~mm}$} & $63.1 \pm 8.9$ & $84.3 \pm 5.3$ & \multirow[t]{2}{*}{$p<0.001$} \\
\hline & $(43.9-79.3)$ & (73.3-93.0) & \\
\hline \multicolumn{4}{|l|}{ Heart } \\
\hline \multirow[t]{2}{*}{$3 \% / 3 \mathrm{~mm}$} & $97.6 \pm 3.9$ & $98.8 \pm 3.5$ & \multirow[t]{2}{*}{$p<0.05$} \\
\hline & $(84.1-100)$ & $(83.8-100)$ & \\
\hline \multirow[t]{2}{*}{$3 \% / 2 \mathrm{~mm}$} & $95.4 \pm 3.8$ & $98.4 \pm 3.5$ & \multirow[t]{2}{*}{$p<0.001$} \\
\hline & $(84.6-99.9)$ & $(83.8-100)$ & \\
\hline \multirow[t]{2}{*}{$2 \% / 2 \mathrm{~mm}$} & $92.6 \pm 4.8$ & $98.4 \pm 3.4$ & \multirow[t]{2}{*}{$p<0.001$} \\
\hline & (82.7-99.0) & $(83.8-100)$ & \\
\hline
\end{tabular}

$$
\begin{aligned}
& \delta=100 \% \times\left(D_{\text {calculated }}-D_{\text {measured }}\right) / D_{\text {measured }} \\
& \delta_{\text {out-of-field }}=100 \% \times\left(D_{\text {calculated }}-D_{\text {measured }}\right) / D_{\text {measured }, C A X}
\end{aligned}
$$

The out-of-field profile calculated by RayStation is more consistent with the measured profile than that calculated by $\mathrm{XiO}$ (Table 6). Because the lung structure of breast IMRT is mainly occupied by out-of-field dose, the predicted 3D GPRs of ipsilateral lung derived from
RayStation are significantly higher than those derived from $\mathrm{XiO}(p<0.001)$ (Table 4$)$. Similarly, the deviations between the predicted and planned ipsilateral lung DVH metrics $V_{5 G y}$ and NTCP derived from RayStation are significantly smaller than those from $\mathrm{XiO}(p<0.001)$ (Fig. 4). These results should be attributable to the accuracy of the low-dose area and the out-of-field dose of RayStation, which comprises a dual-source model and is more sophisticated than $\mathrm{XiO}$ for optimizing beam modeling.

As for PTV, the predicted 3D GPRs of PTV derived from RayStation are significantly lower than those derived from $\mathrm{XiO}$ under the $3 \% / 3 \mathrm{~mm}$ and $3 \% / 2 \mathrm{~mm}$ criteria $(p<0.001)$ (Table 4$)$. The deviations between the predicted and planned PTV DVH metrics $\mathrm{D}_{95 \%}$ and TCP derived from RayStation are significantly larger than those derived from $\mathrm{XiO}(p<0.001)$ (Fig. 4). The steep gradient within the PTV region may be causally related to these deviations. Because the RayStation beam model used in our institution is optimized to a relatively small field of step-and-shoot IMRT compared with the $\mathrm{XiO}$ beam model, the penumbra of the RayStation beam profile was steeper than that of the $\mathrm{XiO}$ beam profile in the tail region (Fig. 5). The cross-plane profiles of the $10 \times 10 \mathrm{~cm}^{2}$ field, which is close to the mean equal square of all 21 plans $\left(8.6 \times 8.6 \mathrm{~cm}^{2}\right.$ field $)$ (Table 1), shows that the $\mathrm{XiO}$ data are more consistent with measured data than the RayStation data on the central region and the penumbra (Table 6). However, the differences in value between the predicted PTV $\mathrm{D}_{95 \%}$ and TCP derived from RayStation and $\mathrm{XiO}$ are substantially small, as shown in Table 5.

Our in-house MGDR software does not require any TPS-like dose calculation engine found in commercial MGDR software such as 3DVH or COMPASS. Our software only adds the local error to the planned dose grid along with photon flux to predict the 3D dose distribution. Regarding inhomogeneity correction, our proposed MGDR method takes into consideration the accuracy attributed by the TPS calculation with the original dose grid. The dose validation of this

Table 5 Comparison of predicted clinically relevant dose-volume indices derived from each TPS (XiO or RayStaion)

\begin{tabular}{llll}
\hline Predicted clinically relevant dose-volume indices & XiO Mean \pm SD & RayStation Mean \pm SD & $P$ value \\
\hline PTV $D_{95 \%}(G y)$ & $47.0 \pm 0.5$ & $46.8 \pm 0.4$ & $p<0.001$ \\
Heart $D_{\text {mean }}(G y)$ & $2.5 \pm 0.9$ & $2.4 \pm 0.9$ & $p<0.001$ \\
Lung $V_{5 G y}(\%)$ & $23.7 \pm 6.4$ & $19.2 \pm 5.8$ & $p<0.001$ \\
Lung $V_{20 G y}(\%)$ & $10.3 \pm 4.8$ & $10.0 \pm 4.6$ & $p<0.005$ \\
TCP of PTV & $0.9199 \pm 0.0034$ & $0.9184 \pm 0.0029$ & $p<0.005$ \\
NTCP of Ipsilateral Lung & $0.0079 \pm 0.0074$ & $0.0062 \pm 0.0061$ & $p<0.001$ \\
NTCP of Heart & $0.0034 \pm 0.0037$ & $0.0035 \pm 0.0037$ & Not significant
\end{tabular}

PTV $D_{95 \%}$ : dose coverage $95 \%$ volume of PTV. Heart $D_{\text {mean }}$ : dose coverage mean volume of Heart. Lung $V_{5 G y}$ or $V_{20 G y}$ : volume receiving at least 5 Gy or 20 Gy of ipsilateral lung 


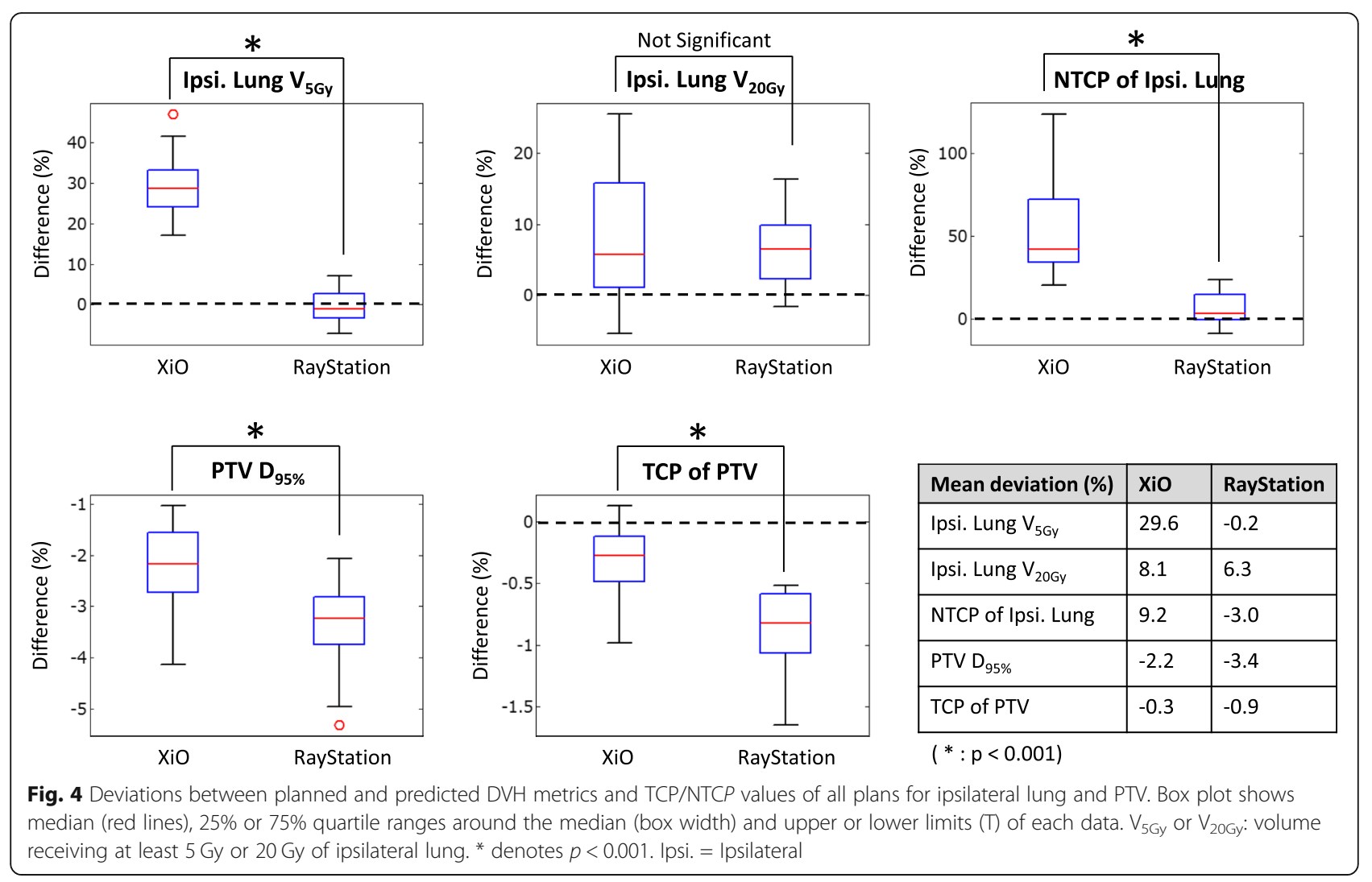

software has been implemented [14]. Therefore, the proposed method is free from errors associated with incorrect recalculations and additional uncertainties.

Note that there are some limitations associated with this study. First, we do not consider the motion-interplay effect. Our previous study [15] showed that motion-interplay results in underdosing to the target. However, because the motion-interplay effect is independent of the calculation process of GPR and MGDR, it is not subject to discussion.

Second, the beam model used for the TPS to calculate and measure data can still be optimized. Table 7 shows the effects of change in leaf tip width, which is one of the parameters of RayStation beam modeling. Chen et al. stated that leaf tip width specifies "the dimension of leaf end which has a transmission factor equal to the square root of average MLC radiation transmission" [23]. Similar to the comparison of the two types of TPSs, the change of beam modeling parameter affects the 2D GPR, the predicted 3D GPR, and the predicted per-organ 3D GPR. Nonetheless, because this study was established to compare GPRs and the predicted clinically relevant dose-volume indices and the difference of indices of the predicted $3 \mathrm{D}$ patient dose distribution derived from the two TPSs, this issue lies outside the scope of this paper.

Recently, the AAPM Medical Physics Practice Guidelines 5.a. recommended " $2 \% / 2 \mathrm{~mm}$, no pass rate tolerance, but areas that do not pass need to be investigated" for the commissioning of IMRT/VMAT dose validation systems in the case of planar or

Table 6 Summary of the average deviation (\%) and distance to agreement ( $\mathrm{mm}$ ) from curve-quality metrics for cross-plane profiles (averaged over all depths: $d_{\max }, 5$, and $10 \mathrm{~cm}$ )

\begin{tabular}{|c|c|c|c|c|c|c|}
\hline \multirow[t]{2}{*}{ field size } & \multicolumn{3}{|l|}{$\mathrm{XiO}$} & \multicolumn{3}{|l|}{ RayStation } \\
\hline & central (\%) & penumbra $(\mathrm{mm})$ & out-of-field (\%) & central (\%) & penumbra (mm) & out-of-field (\%) \\
\hline $2 \mathrm{~cm}$ & $4.36 \pm 4.10$ & $0.97 \pm 0.58$ & $2.75 \pm 2.39$ & $1.20 \pm 1.69$ & $0.51 \pm 0.15$ & $0.47 \pm 0.92$ \\
\hline $5 \mathrm{~cm}$ & $1.27 \pm 2.03$ & $0.95 \pm 0.63$ & $2.21 \pm 2.06$ & $0.54 \pm 1.23$ & $0.52 \pm 0.36$ & $0.49 \pm 0.59$ \\
\hline $10 \mathrm{~cm}$ & $0.37 \pm 0.51$ & $0.51 \pm 0.39$ & $1.39 \pm 1.00$ & $0.49 \pm 1.11$ & $0.61 \pm 0.45$ & $0.57 \pm 0.73$ \\
\hline $20 \mathrm{~cm}$ & $0.34 \pm 0.50$ & $0.50 \pm 0.28$ & $1.52 \pm 0.89$ & $0.34 \pm 0.66$ & $0.82 \pm 0.68$ & $0.79 \pm 0.88$ \\
\hline
\end{tabular}

Square field sizes was $2,5,10$, and $20 \mathrm{~cm}$. Source-surface distance of all data was $100 \mathrm{~cm}$. $d_{\text {max }}$ : depth of dose maximum 

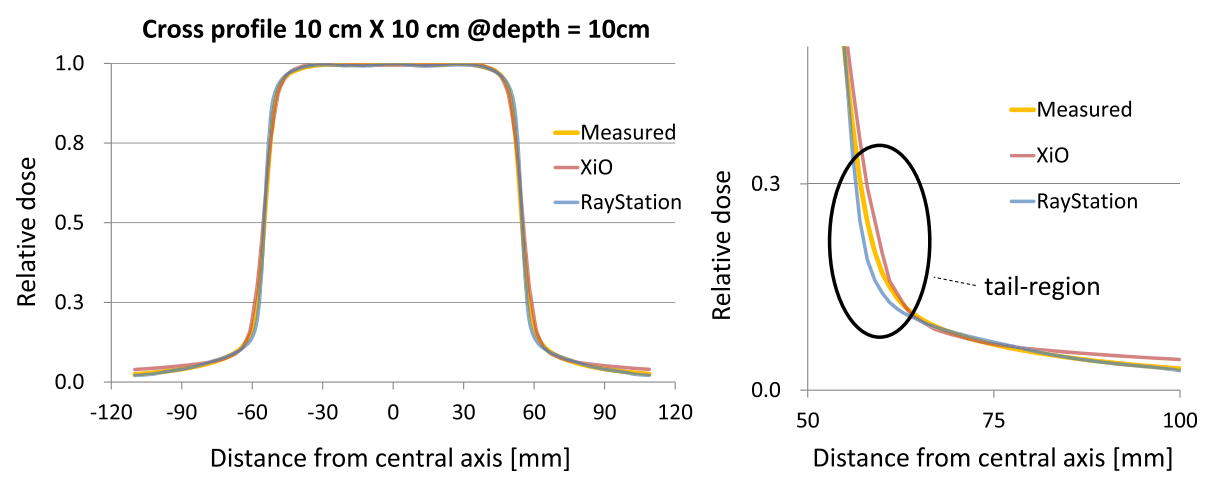

Fig. $56 \mathrm{MV}$ open-field cross-plane profiles measured and calculated by both TPSs for a field size of $10 \mathrm{~cm} \times 10 \mathrm{~cm}$ and a depth of $10 \mathrm{~cm}$, all at a resolution of $0.1 \mathrm{~cm}$ and normalized at the central beam axis

volumetric arrays [36]. In addition, the AAPM TG 218 recommended, "If the plan fails, evaluate the gamma failure distribution and determine if the failed points lie in regions where the dose differences are clinically irrelevant" for gamma analysis [4]. These statements imply the importance not only of increasing the value of GPR but also of discussing where the error is located and what it clinically implies. As described in this paper, the clinically relevant dose-volume indices predicted by MGDR help to understand the effect of dose error clinically on each organ. In addition, when we interpret the predicted 3D patient dose distribution, we should be more aware of the possibility that the results depend on the accuracy of TPS dose calculation.

\section{Conclusions}

In 21 cases of fixed-gantry step-and-shoot breast IMRT, the same DICOM plan was calculated using two different TPSs: $\mathrm{XiO}$ and RayStation. Using in-house software, each predicted 3D dose distribution was derived by referencing each TPS and the measured dose using a planar array detector. Although the mean 2D GPRs calculated by commercial software were $95 \%$ or over under $3 \%$ global $/ 3 \mathrm{~mm}$ criterion, the predicted per-organ 3D GPRs did not correlate with the 2D GPRs under $3 \%$ global $/ 3 \mathrm{~mm}$ criterion. In addition, the predicted 3D GPRs of ipsilateral lung derived from RayStation are significantly higher than those derived from $\mathrm{XiO}(p<0.001)$ under all criteria. The clinical interpretation of these results was explained by the predicted clinically relevant dose-volume indices. The predicted ipsilateral lung DVH metrics $\mathrm{V}_{5 \mathrm{~Gy}}$ and NTCP derived from RayStation were better than those derived from XiO because RayStation has better accuracy regarding calculation of the out-of-field and low-dose-area distribution.

Although we can assume that the GPR can detect dosimetric error for patient-specific QA, in case we require additional clinical and biological consideration of the actual irradiated dose distribution, we could add the predicted clinically relevant dose-volume indices on the presupposition that the accuracy of the dose calculation of TPS is guaranteed.

Table 7 Summary of the 2D GPR, predicted 3D GPR, and predicted per-organ 3D GPR under 2\% global/2 mm criterion in 7 patients in the case of the change of beam modeling parameter of RayStation

\begin{tabular}{llll}
\hline RayStation $(n=7)$ 2\% global/2 mm & Original leaf tip width: 0.1 & leaf tip width: 0.25 & leaf tip width: 0.4 \\
& Mean \pm SD (range) & Mean \pm SD (range) & $98.8 \pm 1.8$ \\
\hline 2D GPR & $99.8 \pm 0.4$ & $99.5 \pm 0.7$ & $(93.2-100.0)$ \\
Predicted 3D GPR & $(98.7-100.0)$ & $(97.9-100.0)$ & $87.4 \pm 2.9$ \\
& $88.9 \pm 1.5$ & $88.4 \pm 2.4$ & $(83.1-90.3)$ \\
Predicted 3D GPR of PTV & $(86.1-90.6)$ & $(84.7-90.5)$ & $80.6 \pm 8.1$ \\
& $88.8 \pm 5.6$ & $85.2 \pm 7.0$ & $(64.7-87.8)$ \\
Predicted 3D GPR of ipsilateral lung & $(76.8-93.2)$ & $(71.3-93.6)$ & $85.7 \pm 5.9$ \\
& $85.7 \pm 5.2$ & $84.8 \pm 5.8$ & $(75.3-93.9)$ \\
Predicted 3D GPR of heart & $(76.6-93.0)$ & $(75.9-93.1)$ & $97.4 \pm 4.1$ \\
& $96.8 \pm 5.8$ & $97.0 \pm 4.9$ & $(88.5-99.7)$ \\
\hline
\end{tabular}




\section{Abbreviations}

AAPM TG: American Association of Physicists in Medicine Task Group; CAX: Central beam axis; CT: Computed tomography; DICOM-RT: Digital Imaging and Communications in Medicine-Radiotherapy; DVH: Dose-volume histogram; GPR: Gamma passing rate; IMRT: Intensity-modulated radiation therapy; MGDR: Measurement-guided dose reconstruction; MLC: Multileaf collimator; OAR: Organ at risk; PTV: Planning target volume; QA: Quality assurance; TCP/NTCP: Tumor-control probability/normal tissue complication probability; TPS: Treatment planning system

\section{Acknowledgements}

The authors would like to thank Enago (www.enago.jp) for the English language review.

\section{Funding}

This work was partially supported by Grant-in-Aid (KAKENHI, 15 K09995) from Japan Society for the Promotion of Science.

\section{Availability of data and materials}

The datasets generated and/or analyzed during the current study are available from the corresponding author on reasonable request.

\section{Authors' contributions}

AK organized and performed the experiments, performed statistical analysis, and drafted the manuscript. IS initiated the project and was involved in the study design, data interpretation and editing the draft as a good mentor of AK. HM, FI, OS, YS, KO and KT were concerned with the patient dose distribution and treatment. KO supervised the overall study. All authors read and approved the final manuscript.

\section{Ethics approval and consent to participate} Not applicable.

\section{Consent for publication}

Not applicable.

\section{Competing interests}

The authors declare that they have no competing interests.

\section{Publisher's Note}

Springer Nature remains neutral with regard to jurisdictional claims in published maps and institutional affiliations.

\section{Received: 20 June 2018 Accepted: 27 January 2019}

Published online: 26 February 2019

\section{References}

1. Li JS, Lin T, Chen L, Price RA Jr, Ma CM. Uncertainties in IMRT dosimetry. Med Phys. 2010;37(6):2491-500.

2. Low DA, Harms WB, Mutic S, Purdy JA. A technique for the quantitative evaluation of dose distributions. Med Phys. 1998;25(5):656-61.

3. Ezzell GA, Burmeister JW, Dogan N, LoSasso TJ, Mechalakos JG, Mihailidis D, et al. IMRT commissioning: multiple institution planning and dosimetry comparisons, a report from AAPM task group 119. Med Phys. 2009;36(11): 5359-73.

4. Miften M, Olch A, Mihailidis D, Moran J, Pawlicki T, Molineu A, et al. Tolerance limits and methodologies for IMRT measurement-based verification QA: recommendations of AAPM task group no. 218. Med Phys. 2018;45(4):e53-83.

5. Coleman L, Skourou C. Sensitivity of volumetric modulated arc therapy patient specific QA results to multileaf collimator errors and correlation to dose volume histogram based metrics. Med Phys. 2013;40(11):7.

6. Stasi M, Bresciani S, Miranti A, Maggio A, Sapino V, Gabriele P. Pretreatment patient-specific IMRT quality assurance: a correlation study between gamma index and patient clinical dose volume histogram. Med Phys. 2012;39(12):7626-34.

7. Nelms BE, Zhen HM, Tome WA. Per-beam, planar IMRT QA passing rates do not predict clinically relevant patient dose errors. Med Phys. 2011;38(2):1037-44.
8. Nelms BE, Opp D, Robinson J, Wolf TK, Zhang G, Moros E, et al. VMAT QA: measurement-guided 4D dose reconstruction on a patient. Med Phys. 2012; 39(7):4228-38

9. Olch AJ. Evaluation of the accuracy of 3DVH software estimates of dose to virtual ion chamber and film in composite IMRT QA. Med Phys. 2012;39(1):81-6.

10. Nelms BE, Simon WE. Radiation therapy plan dose perturbation system and method. U.S. patent 7,945,022 May 17,2011.

11. Zhen $\mathrm{H}$, Nelms BE, Tomé WA. On the use of biomathematical models in patient-specific IMRT dose QA. Med Phys. 2013;40(7):071702.

12. Boggula R, Lorenz F, Mueller L, Birkner M, Wertz H, Stieler F, et al. Experimental validation of a commercial $3 D$ dose verification system for intensity-modulated arc therapies. Phys Med Biol. 2010;55(19):5619-33.

13. Sumida I, Yamaguchi H, Kizaki H, Aboshi K, Tsujii M, Yoshikawa N, et al. Novel radiobiological gamma index for evaluation of 3-dimensional predicted dose distribution. Int J Radiat Oncol Biol Phys. 2015;92(4):779-86.

14. Sumida I, Yamaguchi H, Kizaki H, Aboshi K, Yamada Y, Yoshioka Y, et al. Three-dimensional dose prediction based on two-dimensional verification measurements for IMRT. J Appl Clin Med Phys. 2014;15(5):133-46.

15. Sumida I, Yamaguchi H, Das IJ, Kizaki H, Aboshi K, Tsujii M, et al. Evaluation of the radiobiological gamma index with motion interplay in tangential IMRT breast treatment. J Radiat Res. 2016;57(6):691-701.

16. Hamatani N, Sumida I, Takahashi Y, Oda M, Seo Y, Isohashi F, et al. Threedimensional dose prediction and validation with the radiobiological gamma index based on a relative seriality model for head-and-neck IMRT. J Radiat Res. 2017;58(5):701-9.

17. Ma TJ, Podgorsak MB, Kumaraswamy LK. Accuracy of one algorithm used to modify a planned DVH with data from actual dose delivery. J Appl Clin Med Phys. 2016;17(5):273-82.

18. Miften M, Wiesmeyer M, Monthofer S, Krippner K. Implementation of FFT convolution and multigrid superposition models in the FOCUS RTP system. Phys Med Biol. 2000;45(4):817-33.

19. Valdenaire S, Mailleux H, Fau P. Modeling of flattening filter free photon beams with analytical and Monte Carlo TPS. Biomed Phys Eng Express. 2016;2(3):035010.

20. Deng J, Jiang SB, Kapur A, Li JS, Pawlicki T, Ma CM. Photon beam characterization and modelling for Monte Carlo treatment planning. Phys Med Biol. 2000;45(2):411-27.

21. Liu HH, Mackie TR, McCullough EC. A dual source photon beam model used in convolution/superposition dose calculations for clinical megavoltage x-ray beams. Med Phys. 1997;24(12):1960-74.

22. Mzenda B, Mugabe KV, Sims R, Godwin G, Loria D. Modeling and dosimetric performance evaluation of the RayStation treatment planning system. J Appl Clin Med Phys. 2014;15(5):4787.

23. Chen S, Yi BY, Yang X, Xu H, Prado KL. D'souza WD. Optimizing the MLC model parameters for IMRT in the RayStation treatment planning system. J Appl Clin Med Phys. 2015;16(5):322-32.

24. RayStation 4.5 RayPhysics manual. Stockholm: Raysearch Laboratories. Chapter 5. Beam model parameters for photons; 2014.

25. Gagliardi G, Bjohle J, Lax I, Ottolenghi A, Eriksson F, Liedberg A, et al. Radiation pneumonitis after breast cancer irradiation: analysis of the complication probability using the relative seriality model. Int J Radiat Oncol Biol Phys. 2000;46(2):373-81.

26. Abo-Madyan Y, Aziz MH, Aly M, Schneider F, Sperk E, Clausen S, et al. Second cancer risk after 3D-CRT, IMRT and VMAT for breast cancer. Radiother Oncol. 2014;110(3):471-6.

27. Nielsen TB, Wieslander E, Fogliata A, Nielsen M, Hansen O, Brink C. Influence of dose calculation algorithms on the predicted dose distributions and NTCP values for NSCLC patients. Med Phys. 2011;38(5):2412-8.

28. Hedin E, Bäck A. Influence of different dose calculation algorithms on the estimate of NTCP for lung complications. J Appl Clin Med Phys. 2013;14(5): 127-39.

29. RTOG 1005 Protocol: A Phase III Trial Of Accelerated Whole Breast Irradiation With Hypofractionation Plus Concurrent Boost Versus Standard Whole Breast Irradiation Plus Sequential Boost For Early-Stage Breast Cancer. http:// www.rtog.org/ClinicalTrials/ProtocolTable/StudyDetails.aspx?action= openFile\&FilelD=9366. Accessed 2 Feb 2019.

30. Fraass B, Doppke K, Hunt M, Kutcher G, Starkschall G, Stern R, et al. American Association of Physicists in Medicine radiation therapy committee task group 53: quality assurance for clinical radiotherapy treatment planning. Med Phys. 1998;25(10):1773-829. 
31. Mijnheer BOA, Fiorino C, Hartmann G, Knöös T, Rosenwald JC, Welleweerd H. ESTRO booklet No. 7: Quality assurance of treatment planning systems: practical examples for non-IMRT photon beams. Brussels: ESTRO; 2004.

32. Kim Y, Tomé WA. Risk-adaptive optimization: selective boosting of high-risk tumor subvolumes. Int J Radiat Oncol Biol Phys. 2006;66(5):1528-42.

33. Källman P, Ågren A, Brahme A. Tumour and normal tissue responses to fractionated non-uniform dose delivery. Int J Radiat Biol. 1992;62(2):249-62.

34. Mayles P, Nahum A, Rosenwald J-C. Handbook of radiotherapy physics: theory and practice. Boca Raton: CRC Press; 2007.

35. Venselaar J, Welleweerd H, Mijnheer B. Tolerances for the accuracy of photon beam dose calculations of treatment planning systems. Radiother Oncol. 2001;60(2):191-201.

36. Smilowitz JB, Das IJ, Feygelman V, Fraass BA, Kry SF, Marshall IR, et al. AAPM medical physics practice guideline 5 . A.: commissioning and QA of treatment planning dose calculations-megavoltage photon and electron beams. J Appl Clin Med Phys. 2015;16(5):14-34.

37. Okunieff $P$, Morgan D, Niemierko A, Suit HD. Radiation dose-response of human tumors. Int J Radiat Oncol Biol Phys. 1995;32(4):1227-37.

38. Horton JK, Halle JS, Chang SX, Sartor Cl. Comparison of three concomitant boost techniques for early-stage breast cancer. Int J Radiat Oncol Biol Phys. 2006;64(1):168-75.

39. Gagliardi G, Constine LS, Moiseenko V, Correa C, Pierce LJ, Allen AM, et al. Radiation dose-volume effects in the heart. Int J Radiat Oncol Biol Phys. 2010;76(3):S77-85

Ready to submit your research? Choose BMC and benefit from:

- fast, convenient online submission

- thorough peer review by experienced researchers in your field

- rapid publication on acceptance

- support for research data, including large and complex data types

- gold Open Access which fosters wider collaboration and increased citations

- maximum visibility for your research: over $100 \mathrm{M}$ website views per year

At BMC, research is always in progress.

Learn more biomedcentral.com/submissions 